\title{
The role of breakfast in the diets of school-aged children (5-12Y) in Ireland
}

\author{
Ioana Rusu ${ }^{1,2}$, Laura Kehoe ${ }^{1}$, Maria Buffini ${ }^{3}$, John Kearney ${ }^{4}$, Breige A McNulty ${ }^{3}$, Albert Flynn ${ }^{1}$ \\ and Janette Walton ${ }^{2}$ \\ ${ }^{1}$ School of Food and Nutritional Sciences, University College Cork, Republic of Ireland, \\ ${ }^{2}$ Dept. Biological Sciences, Cork Institute of Technology, Cork, Republic of Ireland, \\ ${ }^{3}$ UCD Institute of Food and Health, University College Dublin, Belfield, Dublin 4, Republic of Ireland and \\ ${ }^{4}$ School of Biological \& Health Sciences, Technological University Dublin, Republic of Ireland
}

Breakfast has been described as the most important meal of the day with studies showing an association between breakfast consumption and improved daily nutrient intakes in children, as well as a lower BMI and higher levels of physical activity ${ }^{(1)}$. It has been proposed that the benefits of breakfast consumption are dependent on the type of foods consumed at breakfast and the type of foods consumed during the remainder of the day ${ }^{(1)}$. The aim of this study was to characterise breakfast in school-aged children in Ireland and to investigate the contribution of breakfast to overall daily nutrient intake.

Analyses were based on the National Children's Food Survey II (NCFSII) (2017-2019) ${ }^{(2)}$. A 4-day weighed food record was used to collect food and beverage intake data from 600 children (5-12y). Nutrient intakes were estimated using Nutritics $\subset$ based on UK and Irish food composition data. Breakfast was self-defined by the participant and reported in their food record. In line with recommendations on breakfast studies, this study confined analyses on breakfast to nutrients derived from breakfast foods only and excluded intakes from nutritional supplements. Mean daily intakes of energy and selected nutrients from breakfast foods only and total diet (including nutritional supplements) were calculated using SPSS V26.

Breakfast was consumed on at least one recording day by $100 \%$ of participants with an uptake of $99 \%$ of potential breakfast occasions. The mean (SD) energy intake at breakfast was $298(110) \mathrm{kcal}$, contributing on average $20 \%$ to overall daily energy intake. Energy intake from breakfast comprised of 59\% carbohydrate, $28 \%$ protein and $14 \%$ fat. The most frequently consumed foods at breakfast included 'breakfast cereals with milk' (mainly ready-to-eat breakfast cereals), 'breads' (white/brown/wholemeal), 'table sugar \& sweet spreads', 'butter \& fat spreads' and 'fruit'. The most frequently consumed beverages included 'water' and 'fruit juices'. The contribution of breakfast to daily nutrient intakes was $22 \%$ for carbohydrate, $18 \%$ for protein and $16 \%$ for total fat. Breakfast also contributed to total daily intakes of dietary fibre $(22 \%)$, B-vitamins $(22-40 \%)$, vitamin C (21\%), vitamin D (34\%), vitamin E $(16 \%)$, calcium $(30 \%)$ and iron $(33 \%)$ but made contributions to intakes of saturated fat $(18 \%)$, total sugars $(24 \%)$ and free sugars $(23 \%)$.

Breakfast was widely consumed among school-aged children in Ireland and was typically a nutrient-dense meal that contributed significantly to total dietary intakes of a number of important macro- and micro- nutrients. Relative to its contribution to energy, breakfast contributed to higher proportions of key micronutrients such as thiamin, riboflavin, vitamins B6, B12, folate, vitamin D, calcium and iron while also contributing to considerable proportions of total and free sugars.

\section{References}

1. C. E. O’Neil, T. A. Nicklas and V. L. Fulgoni (2015). AIMS Public Health, 2(3), 441-468.

2. Irish Universities Nutrition Alliance (IUNA) (2019). The National Children's Food Survey II (NCFS II). Available: https://www.iuna.net/ surveyreports. 